\title{
Effect of combining different photoperiod regimes on the sexual maturation of male sea bass (dicentrarchus labrax I.) during the first two years of life
}

\begin{abstract}
This study assesses the effect of combining different photoperiod regimes on the sexual maturation and growth of male sea bass during the first two years of life. In two different trials, regimes of continuous light (LL) lasting 24 consecutive months (Trial 1) or shorter periods within the summer-early autumn months during the first and second annual cycles (Trial 2) were compared to control groups under a natural photoperiod (NP). In a third trial (Trial 3), fish kept under either NP or LL conditions during the first year were subsequently assigned a treatment involving exposure to NP or a constant long photoperiod (LP) throughout the second year. The rates of spermiating males, gonadal size (GSI) and growth under all these photoperiod regimes were analyzed. Exposure to LL reduced the rates of precocity in all trials $(<3 \%)$ at the end of year one as compared to NP $(13-22 \%)$, but full spermiation was still observed in year two (Trials 1 and 2), indicating that LL is not efficient for blocking gametogenesis at this time. Fish exposed to LP in Trial 3 displayed high GSI values (April) and a significant delay in gonadal maturation (62.50-100\%; May) with respect to those exposed to NP (12.5\%), which enhances the potential growth of the fish. In summary, exposure to LL reduces precocity in underyearling fish, while LP delays maturation during the second year, thus providing a promising approach for controlling male sea bass reproduction and growth.
\end{abstract}

Keywords: Day length, Precocity, Puberty, Growth, Reproduction
Volume 5 Issue 4 - 2017

\author{
Alicia Felip, Carrillo M, Rodr guez R, Zanuy S \\ Consejo Superior de Investigaciones Cient
}

Correspondence: Alicia Felip, Consejo Superior de Investigaciones Cient, Tel 34-964-319500, Emailm.carrillo@csic.es

Received: March 01, 2017| Published: April 18, 2017

\section{Introduction}

Control over reproduction is one of the major challenges in the fish farming of commercial aquaculture species, as it is crucial for improving fishery production. Numerous studies have provided relevant information related to genetic, hormonal and environmental approaches for controlling sexual maturation in farmed finfish. ${ }^{1-3}$ In particular, environmental tools based on manipulating the photoperiod have been investigated, demonstrating their potential application in aquaculture. ., $, 5,6$ However, since differences between indoor and outdoor conditions can exist, their utility on a commercial scale needs to be examined..$^{7,8} \mathrm{~A}$ large number of environmental factors are known to potentially control reproduction in most temperate fish, including sea bass. In this regard, while temperature can affect reproductive development, photoperiod is thought to be the main environmental cue for many seasonal breeders. ${ }^{1,4}$ In fact, it is well documented that photoperiod plays an important role in the control of gonadal maturation in farmed fish species such as salmonids, gadoids, breams, bass and flatfish. ${ }^{5,4,6}$

The European sea bass (Dicentrarchus labrax L.) is a highlyprized marine teleost fish that in Mediterranean areas reaches puberty at 2 years of age in males, and at around 3-4 years in females under natural conditions. ${ }^{7,9}$ However, under intensive culture conditions, a non-negligible number of under yearling males (i.e., 20-30\% of the population) exhibit precocious maturation, which negatively affects their farming and commercialization. ${ }^{5,9}$ Although data are still limited, precocity also seems to affect farmed females (S. Zanuy and M. Carrillo, personal communication). This is an especially critical situation, since farmed sea bass often show skewed sex ratios, with $70 \%$ (or more) of males exhibiting $20-40 \%$ lower body weight at harvest. ${ }^{5,9,10}$ Although precocious males are larger in size and have higher GSI values during their first year of life, they show a reduced body size with respect to their counterparts 75 over their second year of life..$^{11,12}$ The effect of photoperiod on sexual maturation, somatic growth and hormonal aspects has been studied in this species for more than three decades now. ${ }^{7,11,13-21}$ Recently, the analysis of the effect of discrete windows of continuous light (LL, $24 \mathrm{~h}$ light/day) on sexual maturation has shown that $85 \mathrm{LL}$ regimes reduce precocious gonadal maturation in juvenile male sea bass during their first year of life..$^{5,11,19,20,21}$ Interestingly, it has been confirmed that including the month of September in the LL this time interval is crucial for reducing precocious gametogenesis in male sea bass, as this is considered to be the most sensitive photolabile period in this species. ${ }^{21}$ Furthermore, it is known that administration of a constant long photoperiod (LP) (15L:9D; light: darkness) delays the onset of puberty in sea bass. ${ }^{9,15,17,18}$ Consequently, the combination of different photoperiods might be a useful environmental strategy to control gonadal development in fish. This is supported by previous studies on male sea bass examining the influence of day length on a number of physiological variables during the first two or three $^{17}$ years of life. One aim of this study was to explore the effect of LL both at year one (already well documented) and at year two, the time at which the onset of male puberty generally takes place under natural conditions. The effect of LL at this time is completely unknown, although it is a very important aspect to be considered for the environmental control of reproduction and for assessing the likely existence of an endogenous reproductive component. The second aim was to monitor gonadal growth during the first two annual cycles of life in male groups kept under different combinations of photoperiod 
regimes to determine their capacity to reduce the rates of precocity at year one exposure to $\mathrm{LL}^{11,20,21}$ and delay the onset of puberty at year two exposure to LP. ${ }^{14,15,17,18}$ To that end, three independent trials were set up to explore, under laboratory conditions, the effect of day length and natural conditions of temperature on growth performance, gonadal size and the rates of running males up to the age at which fish reach a commercial size (450-500 g) and are harvested. In Trial 1 (T1), underyearling male sea bass were exposed to NP (control) or LL for 24 consecutive months to study the inhibitory effect of continuous light on gonadal development during the first and second annual cycle. Trial 2 (T2) used the same experimental design as Trial 1 , except that continuous light was replaced by two shorter exposures to LL (4 months (year one) and 2.5 months (year two) in duration) within the sensitive photolabile period in the summer-early autumn, ${ }^{21}$ and the results were then compared to those of T1. This is of particular interest in commercial operations, because if reduced lighting were able to impair sexual maturation, it would help minimize the stress on animals and would reduce farming costs by providing for optimal yields in fish farms. Finally, in Trial 3 (T3), fish were exposed to either NP or LL conditions for 12 consecutive months during the first annual cycle (i.e., year one). Subgroups of these groups were then exposed to either NP or LP during year two, in order to evaluate the effect of the photoperiod regimes applied on delaying the onset of puberty and enhancing growth at the age of commercialization.

\section{Materials and methods}

\section{Fish and experimental conditions}

All juvenile male sea bass (2-4 g) were purchased from Aquanord (Gravelines, France) and distributed into separate identical 2000 L light-proof circular fiberglass tanks provided with well-aerated running sea water (salinity $=37-38 \%$ ) at the Instituto de Acuicultura de Torre la Sal (Castellón, Spain, $40^{\circ} \mathrm{N} ; 0^{\circ} \mathrm{E}$ ) facilities. Temperature naturally ranged between $12-25 \pm 1{ }^{\circ} \mathrm{C}$. The feeding regime was adjusted according to temperature and fish size, based on standard procedures. ${ }^{22}$ Different photoperiod regimes were combined and implemented in three independent trials, in order to study the effect of photoperiod on gonadal growth during two consecutive annual cycles: the incipient reproductive period (first annual cycle; year one) and the puberty (second annual cycle; year two), ${ }^{7}$ Somatic growth was evaluated during the second annual cycle, coinciding with the full reproductive season of male fish (Figure 1). ${ }^{7}$

The first trial (T1) compared two experimental groups $(\mathrm{n}=810$ fish; 405 fish/tank): one group exposed to a constant simulated natural photoperiod (NP) and the other maintained under constant continuous light (LL) throughout the first and second annual cycles (Figure 1A). The second trial (T2) also included two groups (n= 180 fish; 90 fish/ tank). The first group, which acted as a control, was exposed to NP during the entire first and second annual cycles. The second group, on the other hand, was maintained under LL within two different time windows: from June 1 to October 1 (LLJO for 4 months) during the first year and from August 15 to October 31 (LLAO for 2.5 months) during the second year, and otherwise NP during the remaining periods (Figure 1B). This latter group is referred to as LLJO-AO throughout this study. Of note, both LL treatments applied in this trial included the month of September, as this time interval was identified as the most sensitive photolabile period in this species. ${ }^{21}$ In the third trial (T3, n= 348 fish; 174 fish/tank), one group was maintained under NP and a second group under LL conditions during the first year. Coinciding with the resting period (April) of the first annual reproductive cycle of animals, each fish group was equally distributed into two subgroups, which were subsequently exposed to either a natural light cycle (NP) or a constant long photoperiod (LP) with a natural day length of 15L:9D until the end of the experiment, which coincided with the end of the second annual reproductive cycle (Figure 1C). Thus, there were a total of four groups $(\mathrm{n}=330$ fish; 80-82 fish/tank): NP-NP, NP-LP, LL-NP and LL-LP, according to the photoperiod regime they were exposed to during the first and second experimental phases. Artificial light regimes were regulated by means of electronic timers that controlled tungsten bulbs $(650-700$ lux; Philips, PAR38Pro) located at the water's surface. Fish were fed to apparent satiety by automatic feeders that administered pellets from Proaqua Nutrición S.A. (Palencia, Spain) (protein 54-45\%, lipids 20$12 \%$, carbohydrates $9-25 \%$, ash $11 \%$, moisture $1-3 \%$, DE $22.4-19.7$ MJ kg-1).

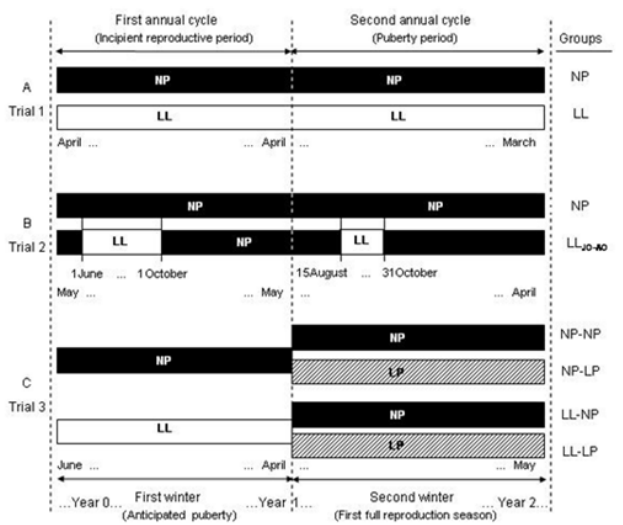

Figure I Schematic representation of the experimental designs and photoperiod regimes for the three independent trials conducted in this study. Trial I (A) compared exposure to a constant simulated natural photoperiod (NP) versus constant continuous light (LL) during two consecutive annual cycles. Trial 2 (B) compared exposure to NP during two consecutive annual cycles with exposure to continuous light from June I to October I (first annual cycle) and from August 15 to October 3I (second annual cycle) under otherwise NP conditions (LLJO-AO). Trial 3 (C) compared four groups exposed to either NP or LL during the first annual cycle, followed by exposure to either NP or a constant long photoperiod of I5L:9D (LP) during the second annual cycle.

\section{Sampling protocol}

To evaluate growth performance during the second annual cycle of life (i.e., year two), fish were anesthetized with 2-phenoxyethanol $(0.5 \mathrm{~mL} \mathrm{~L}-1$ of seawater) before sampling. Each month, the animals from each tank were weighed (W) and measured (L; fork length), and a condition factor was calculated as 100xWL-3. Specific growth rates for weight (GW) and length (GL) were calculated in each sampling as $100(\operatorname{lnWf}-\ln W i) t-1$ and $100(\operatorname{lnLf}-\operatorname{lnLi}) t-1$, respectively, where Wf and $\mathrm{Lf}$ are the final weight $(\mathrm{g})$ and length $(\mathrm{cm})$, Wi and $\mathrm{Li}$ the initial weight and length, and $t$ is the number of days between samplings. Gonad weight was measured in sampled fish from $\mathrm{T} 1$ and $\mathrm{T} 3$, and the gonadosomatic index (GSI) was calculated according to the formula: gonad weight/body weight x 100 . Animals were sacrificed in accordance with the guidelines for animal experiments established by European legislation (ETS No. 123, 01/01/91). Males were periodically stripped in a gentle manner to evaluate spermiation in each trial and calculate the percentage of spermiating males during the first two years of life. Moreover, fish in T2 were gently massaged approximately every fifteen days to record the first spermiation time of individual animals and the cumulative percentage of spermiating 
fish from the beginning of the experiments on, as well as the duration of spermiation (in days) during the second annual cycle.

\section{Statistical analysis}

A one-way ANOVA test was used to compare the effects of the different photoperiod regimes on growth performance and GSI. When necessary, normality was determined by means of a KolmogorovSmirnoff test after the logarithmic transformation of data. Barlett's test was used to establish homogeneity of variances. Tukey's HSD multiple range test was used to examine significant differences between means. Nominal data on the percentage of spermiating fish versus non-spermiating fish under the different photoperiod regimes were compared using the Chi-Square statistic and applying the Yates correction, when $\mathrm{v}=1$, or a Bonferroni inequality for $\mathrm{P}$ values when applying the multiple range test. A linear regression analysis was conducted to determine the relationships between the condition factor and GW, and between the percentage of spermiating males and GL, taking into account the results of all three trials in the present study. All data were expressed as mean \pm SEM. Differences were accepted as significant when $\mathrm{P}<0.05 .^{23}$

\section{Results}

\section{Growth}

In order to evaluate the influence of combining different photoperiod regimes on the somatic growth of 2-year-old males over a two-year period, values for weight, length and condition factor were recorded in all three trials (Figure 2). Specific increases in weight and length are shown in Fig 3. Two different periods could be distinguished in the second annual cycle of life: a period of active somatic growth (May-June to November) and a period of reduced growth (November to April-May), when male sea bass are known to reach puberty (i.e., full gonadal development). The results show that the curve of weight and size increase in LL fish (i.e., LL group in T1 and LLJO-AO group in T2) throughout this time interval had a similar shape to that of their respective NP control groups. However, it is interesting to note that LL groups exhibited significantly lower size values $(\mathrm{P}<0.05)$ than the corresponding NP groups throughout the entire experiment, and as a result the size of the control groups (NP) at 17-18 months of age was significantly larger than that of the LL and LLJO-AO groups (Figure 2A-B \& Figure 2D-E). Differences were particularly marked during the period of active somatic growth in both $\mathrm{T} 1$ and $\mathrm{T} 2$. After 25-26 months of rearing, the mean values for weight and length of the NP group in $\mathrm{T} 1$ were $451.78 \pm 12.46 \mathrm{~g}$ and $31.71 \pm 0.27 \mathrm{~cm}$, as compared to $414.81 \pm 8.68 \mathrm{~g}$ and $30.79 \pm 0.21 \mathrm{~cm}$ in the LL group (T1) (Figure $2 \mathrm{~A}-\mathrm{B})$. In T2, the mean values for the NP group were $483.99 \pm 19.71$ $\mathrm{g}$ and $31.32 \pm 0.36 \mathrm{~cm}$ versus $434.39 \pm 10.62 \mathrm{~g}$ and $30.44 \pm 0.27 \mathrm{~cm}$ for the LLJO-AO group (Figure 2D-E). Overall, this represented an $8-10 \%$ and a $3 \%$ decrease in weight and length, respectively, in the LL groups with respect to the control groups. At the end of this period (approximately 27-28 months of age), significant weight differences were observed between the NP and LL groups, with the LL group exhibiting higher values than the NP group in T1 (Figure 2A), but lower ones in T2 (Figure 2D). During the second half of the full gonadal development period, no significant differences were observed in length between groups for either T1 or T2 (Figure 2B-E). In T3, the differences among groups were particularly noticeable at the end of the full gonadal development period (31 months of age). The mean weight and length values at this time point were $624.24 \pm 15.03 \mathrm{~g}$ and $35.63 \pm 0.25 \mathrm{~cm}$ for the NP-NP group (control), $668.39 \pm 27.67 \mathrm{~g}$ and $36.19 \pm 0.44 \mathrm{~cm}$ for the NP-LP group, $562.98 \pm 19.14 \mathrm{~g}$ and $34.59 \pm$
$0.33 \mathrm{~cm}$ for the LL-NP group, and $576.63 \pm 19.12 \mathrm{~g}$ and $34.69 \pm 0.32$ $\mathrm{cm}$ for the LL-LP group (Figure 2G-H). This represented a weight and length increase of $2.4-7 \%$ and $0.3-1.5 \%$, respectively, for those groups exposed to LP regimes, as compared to those exposed to NP during the second annual cycle. It is important to note that the potential stressful impact of exposing the fish to LL throughout the first year might influence their growth during the second year, thus masking the stimulatory effect of LP on growth during that period. Interestingly, the NP-LP group, which was not exposed to LL at anytime during the study, showed enhanced growth during the second year, which is when the animals were exposed to LP (Figure 2G-H) arrows. In order to assess more precisely the effects of photoperiod on sea bass growth, we analyzed the specific growth rate, which refers to the growth of a given group of fish over a fixed time interval. Overall, our results showed a strong increase in the specific growth rates of weight and length in all three trials between June-July and November, as compared to the values observed during the full gonadal development period. In each trial, the experimental group and the control group kept under simulated natural light (NP) showed a similar pattern of specific growth rate, although the maximum values achieved by both groups differed between trials. In this sense, the highest GW (around $1.2 \%$ ) of all trials in the second active growth period was seen in $\mathrm{T} 2$ during the July-August time interval for both the NP and LLJO-AO groups (Figure 3C). GW peaked (0.71-0.81\%) in June-July for all T3 groups (Figure 3E), and also for both NP and LL groups in T1 (Figure $3 \mathrm{~A})$, albeit with lower values $(0.4 \%)$. Accordingly, differences in the maximum GW averages in this study were as follows: $\mathrm{T} 2>\mathrm{T} 3>\mathrm{T} 1$. On the other hand, the maximum specific growth rate for length was achieved during the September-October time interval in the case of T1 (GL $>0.80 \%$ for NP and LL groups) (Figure 3B). The average value of GL in T2 was $>0.30 \%$ in August-September for both the NP and LLJO-AO groups (Figure 3D), while all groups in T3 showed values of around $0.23-0.28 \%$ GL in July-August (Figure 3F). Accordingly, maximum GL averages were recorded in T1, while those of T2 and T3 were similar to one another. In contrast to the period of active somatic growth, the full gonadal development period was characterized by a reduction in the specific growth rate of weight and length in all groups of every trial (Figure 3). Specific growth rate was higher in LL versus NP groups at some sampling points during the full gonadal development period, while high specific growth rates were recorded in LP groups after 29-31 months of rearing (March-May), which evidenced the growth enhancing effect of LP on sea bass (Figure 3EF). The pattern of variation for the condition factor in all three trials is depicted in Fig. 2. It can be seen that trials which displayed high condition factor values also had elevated GW values. Consequently, the fish in T2, which had greater GW values than those in T3 and T1, also reached the highest condition factor values (Figure 2F) during the second annual cycle. Overall, the variations in maximum average condition factor values in all three trials were in line with those previously observed for $\mathrm{GW}(\mathrm{T} 2>\mathrm{T} 3>\mathrm{T} 1)$. $\mathrm{CF}$ values for the control groups in all three trials (NP in T1 and T2, and NP-NP in T3) were plotted as a function of GW and a regression line was fit to the data points to estimate the increase in $\mathrm{CF}$ according to changes in GW. The values of the determination coefficients $(R 2=0.933$ and $R 2=0.985)$ evidenced a strong association between both variables (Figure 4A).

\section{Gonadomatic index}

Monthly changes in GSI values for the NP and LL groups in T1 were seen from November to March during the second annual cycle (Figure 5A). In November and December, GSI was similar in both groups. The first significant GSI increase in the NP group occurred in 
January and February, when it reached values $>4.7 \%$, only to slowly decrease in March. The LL group had significantly lower values than the NP group in January (3.45\%) and February (3.75\%), and proceeded to slowly decrease in March. GSI values for the NP-NP group in T3 were high in March, and slowly decreased in April and May (Figure 5B). A similar pattern of variation was observed in the LL-NP group. The NP-LP group had a GSI of $1.66 \%$ and $3.38 \%$ in March and April, respectively, which then decreased in May to $0.75 \%$. The LL-LP group also showed high values in March and April (2.76$2.71 \%)$ that again decreased in May $(0.90 \%)$. Of note, both groups kept under LP conditions (i.e., NP-LP and LL-LP) during the second annual cycle showed the highest GSI values in April, at which time the GSI of the NP-NP and LL-NP groups had already started to show a progressive decrease. No data were recorded in $\mathrm{T} 2$.

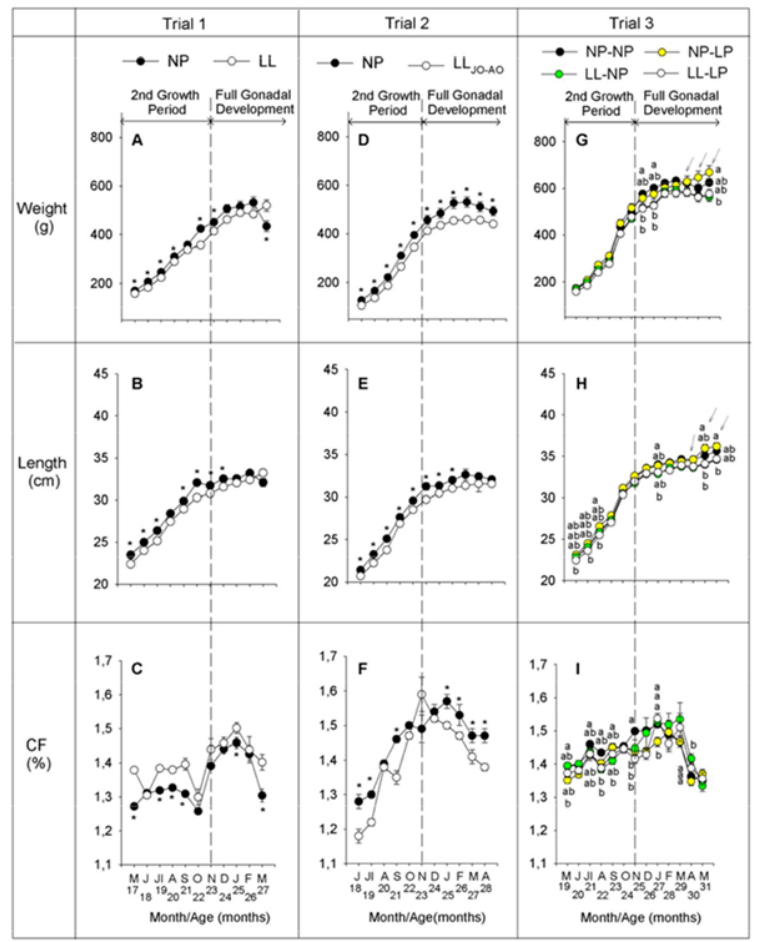

Figure 2 Monthly changes in mean ( \pm SEM) body weight (A,D,G), fork length $(B, E, H)$ and condition factor $(C, F, I)$ during the second annual cycle for male sea bass kept under the different photoperiod regimes described in Figure I. Dotted lines divide this cycle into two different periods, one of active somatic growth (May-June to November) and one of reduced growth (November to April-May), at which time male sea bass are known to reach puberty (i.e., the period of full gonadal development). Significant differences $(P<0.05)$ between photoperiod treatments for the same month are indicated by asterisks in $\mathrm{TI}$ and T2, and different letters in T3. Arrows indicate a potential growth enhancement.

\section{Spermiation rate}

The percentage of running males in all three trials during two consecutive annual cycles is depicted in Fig. 6. The highest GL values, which were seen in T1, correlated with high rates of precocity, while the lower GL values seen in T2 and T3 were associated with lower rates of precocity. A regression line was calculated to estimate the increase in the rate of spermiating males based on the fish GL; there was a strong association between both variables as indicated by the determination coefficient (R2=0.790) (Figure 4B). The percentage of precocious males during the first annual cycle was high in February
$(21.89 \%)$ in the NP group of T1, but steadily decreased in March (14.69\%) and April (2.63\%) (Figure 6A). The LL group showed a very low rate of running fish in March (3.32\%) and April (2.25\%). These results greatly differ from the percentages obtained during the second annual cycle for both the NP and LL groups, which exhibited a similar rate of spermiating fish from November to March, although it should be noted that generally higher rates of spermiation were recorded for the NP group as compared to the LL group (Figure 6B). In this manner, the percentage of maturing males increased throughout this period, peaking in February for both the NP (100\%) and LL (95.65\%) groups, and remaining high until March $(100 \%$ and $92 \%$, respectively, $\mathrm{P}<0.05$ ) (Figure 6B). In T2, the percentage of precocious males in the NP group was as low as $6.5 \%$ in February, while no running fish were observed in the LLJO group at this sampling point of the first annual cycle (Figure 6C). On the other hand, the percentages obtained during the second annual cycle (November-May) for both the NP and LLJO$\mathrm{AO}$ groups were similar, although as was the case in T1, significantly higher rates of spermiation were also recorded in T2 for the NP versus the LLAO (LLJO-AO) group (Figure 6D). Likewise, the highest percentage of spermiating males for both the NP $(95.56 \%)$ and LLJOAO (83.51\%) groups was attained in February, and was maintained from March to May in both groups. Finally, the NP group of fish in T3 exhibited the highest percentage of precocious males in March $(13.10 \%)$, and the lowest percentage in June $(0.90 \%)$ during the first annual cycle, while a very low rate of running males was observed in the LL group from February to June $(0 \%, 3.15 \%, 1.30 \%, 1.90 \%, 0 \%$, respectively) (Figure 6E) $(\mathrm{P}<0.05)$. This greatly contrasted with the percentages (March-May) obtained during the second annual cycle (Figure 6F). Of note, the NP-NP and LL-NP groups displayed the highest percentage of spermiating males (100\%) in March-April and April, respectively, while this rate decreased to $12.50 \%$ in May for both groups. In the two groups kept under LP conditions during the second annual cycle, the highest percentages of spermiating males $(100 \%)$ were found in April. Interestingly, the rates of spermiating fish in May were as high as $62.50 \%$ in the NP-LP group and $100 \%$ in the LL-LP group, in stark contrast with the low percentages $(12.50 \%)$ observed in the NP-NP and LL-NP groups during the same period. These findings show that gonadal maturation in fish exposed to LP was delayed with respect to those kept under natural photoperiod (control) conditions $(\mathrm{P}<0.05)$ throughout the second annual cycle.

\section{Duration of spermiation in males}

In $\mathrm{T} 2$, a low percentage of males in both the NP $(2.2 \%)$ and LLAO (LLJO-AO) $(2.60 \%)$ groups began to release sperm from midNovember on, and the highest percentages of running males were observed at mid-January $(35.53 \%$ and $30.26 \%, 15$ respectively). At later sampling points, this percentage decreased in both groups, with the LLAO (LLJO-AO) group exhibiting the lowest values. Males that released sperm for the first time during the second annual cycle after gentle stripping were still found in both groups at the end of February (4.44\% and $3.90 \%$ in NP and LLAO (LLJO-AO), respectively) (Figure 7A). These results were used to calculate the cumulative percentage of spermiating fish (Figure 7B). The results showed that male sea bass had a rather long reproductive season, with a very low rate of fish spermiating early in November and a steady and progressive increase in the cumulative percentage from December to the end of February (95.56\% in NP and $83.51 \%$ in LLAO group). Constant elevated values were maintained in the following months, until the end of May (Figure 7B). This would indicate that sea bass males were actively spermiating for around 5.9 months. We also observed a few males which started to spermiate at the end of February, i.e., 3.3 months later than those that started in November. Spermiation in the NP and 
LLAO (LLJO-AO) groups of fish lasted from 15 to 180 days, with an average span of 124 days in the NP group and 127 days in the LLAO (LLJO-AO) group (Figure 7C).

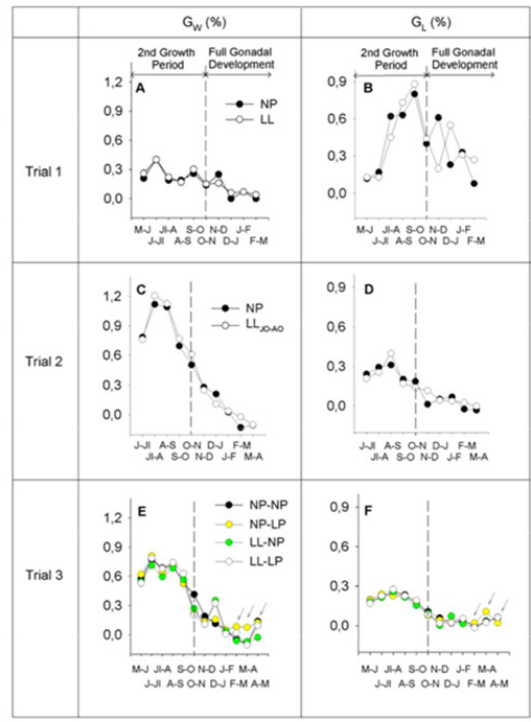

Figure 3 Specific growth rates for body weight (GW) (A, C, E) and fork length $(G L)(B, D, F)$ during the second annual cycle for male sea bass kept under the different photoperiod regimes described in Figure I. Dotted lines divide this cycle into two different periods as explained in Figure 2. Data are expressed as mean values for each experimental group. Arrows indicate a potential growth enhancement.
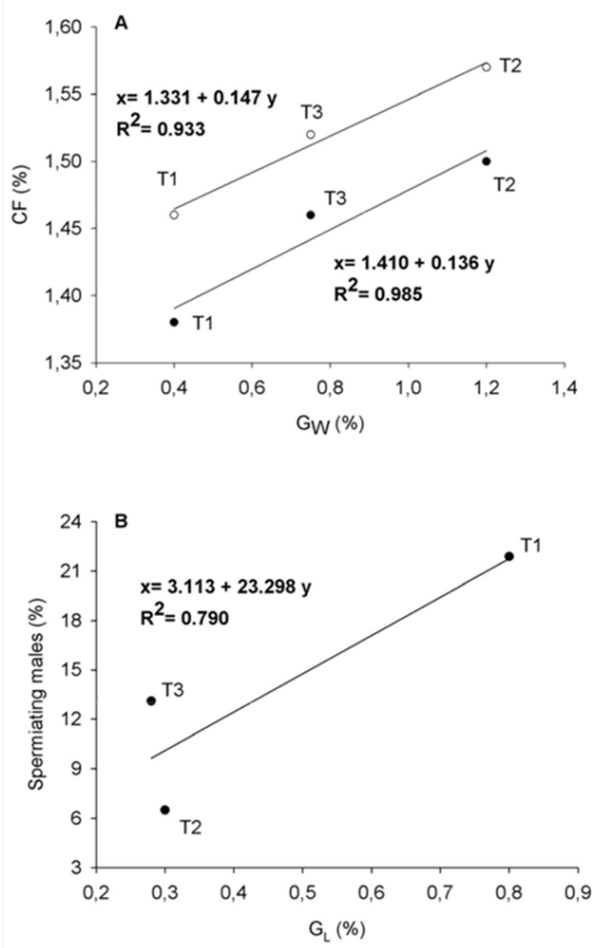

Figure 4 (A) Linear regression of male sea bass condition factor (CF) versus specific growth rate for weight (GW) during the second year growth period (black circles) and full gonadal development (white circles). (B) Linear regression of male sea bass rate of precocity versus specific growth rate for length (GL) during the second year. Data points are taken from all three trials (TI, T2 and T3), taking into account the maximum values recorded in the control groups (NP in TI and T2 and NP-NP in T3) for each variable.
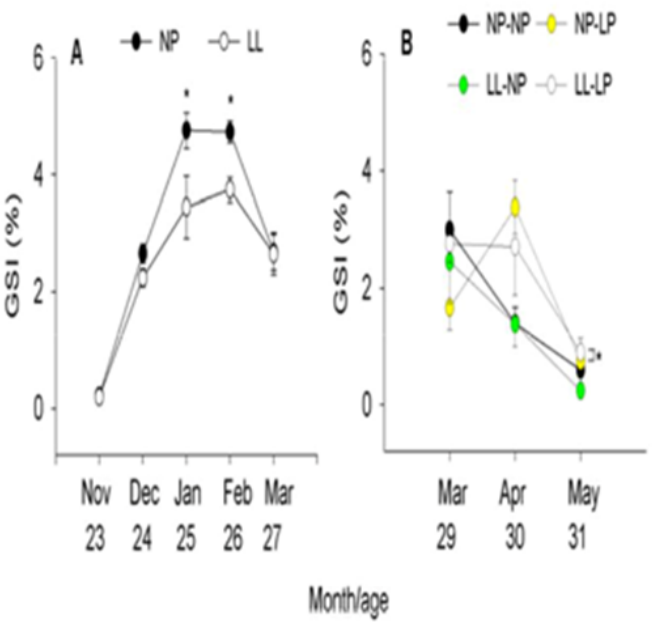

Figure 5 Monthly changes in gonadosomatic index (GSI) mean $( \pm$ SEM) values during the second annual cycle of life of male sea bass kept under different photoperiod regimes in trial I (A) and trial 3 (B). Asterisks indicate significant differences $(P<0.05)$ between photoperiod treatments in the same month. See Figure I for details on the light regimes used.

\section{Discussion}

This paper examined the effect of combining different photoperiod regimes on sexual maturation and growth performance of male sea bass during the first two years of life. Accordingly, our results demonstrate that exposing underyearling male sea bass to ambient seasonal temperatures and LL regimes for either 12 consecutive months (T1 and $\mathrm{T} 3$ ) or during the summer-early autumn period (i.e., from June to October; T2) of the first annual cycles equally effective for reducing the numbers of precocious fish. These LL treatments are just as efficient as those previously applied by Begtashi et al., ${ }^{11}$ who used a 12-month exposure to LL, and Felip et al., ${ }^{20}$ who studied 4-16 and 6-month exposures before and during gametogenesis, respectively. Moreover, the present data confirm the existence of a photolabile period in sea bass that includes the month of September, during which time the application of LL reduces the number of prepubertal males that successfully carry out the process of gametogenesis. ${ }^{21}$ In fact, it is known that exposure to LL outside this photolabile period fails to prevent the presence of precocious males, as the administration of LL from October to November during the first year of life had no effect on the percentage of precocious males as compared to the simulated natural photoperiod. ${ }^{21}$ Altogether, this suggests that successful reduction in the occurrence of precocious males would depend on the time of the year when the under yearling fish are exposed to a specific photoperiodic signal. ${ }^{24,17}$ In our study, exposure to LL drastically reduced the percentage of precocious males in all three trials, reaching levels below 3\%, whereas in the control groups these levels were as high as $13-22 \%$ (see also Carrillo et al.. ${ }^{59}$ However, it is interesting to note that differences in the rate of precocious males were also evident among the control groups of different trials. In this study, the maximum rate was recorded in $\mathrm{T} 1$, followed by $\mathrm{T} 3$ and $\mathrm{T} 2$. The study by Rodríguez et al. ${ }^{21}$ hypothesized that populations with larger-size males would also exhibit higher rates of precocity. Our data would support this idea, as T1 groups had both larger fish (GL) and higher rates of precocious fish than T3 and T2 groups $(21.89 \%, 10.60 \%$ and $6.5 \%$, respectively). 


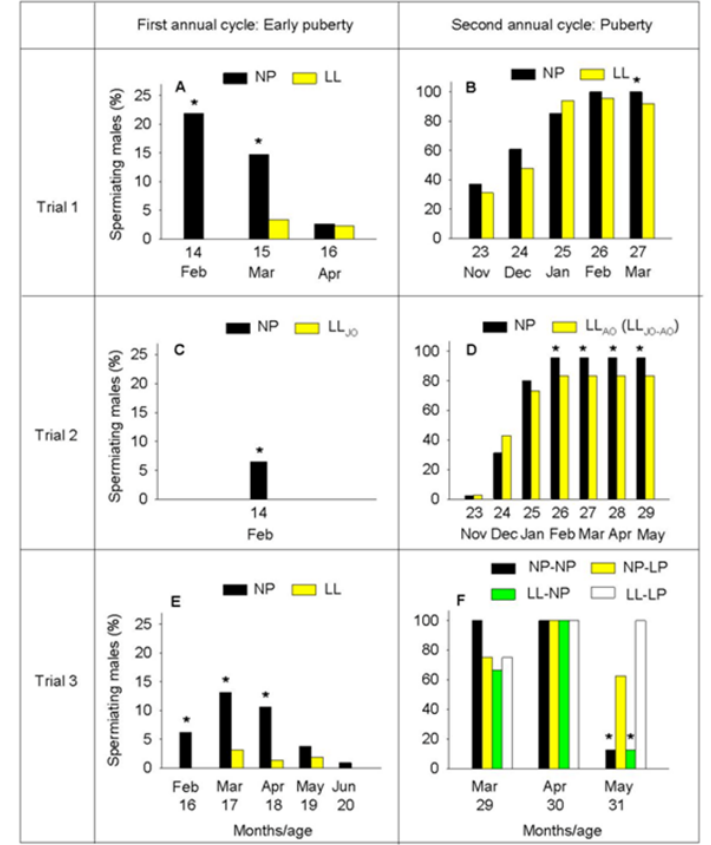

Figure 6 of running sea bass males (as assessed by stripping) kept under the different photoperiod regimes described in Figure I, during the first $(A, C, E)$ and second annual reproductive cycles $(B, D, F)$. The light regime references are explained in Figure I.Asterisks indicate differences between different light regimes in the same month.
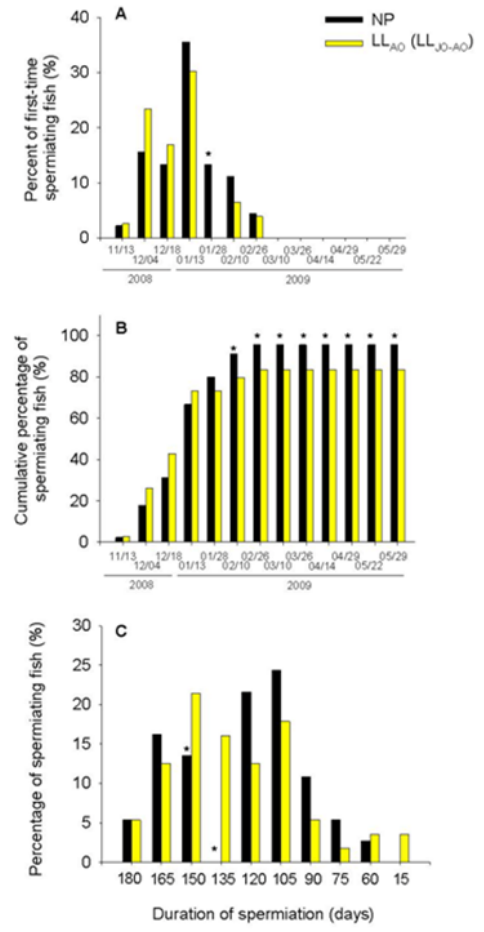

Figure 7 Traits of the maturation process in male sea bass during the second annual cycle. (A) Percentage of males (as assessed by stripping) that started running from mid-November to the end of February. (B) Cumulative percentage of fish spermiating from November to February that remained spermiating until the end of the spawning period (May). (C) Duration of spermiation in sea bass previously kept under different photoperiod regimes (Trial 2). The light regime references are explained in Figure I. Data are expressed as mm-dd-year. Asterisks indicate differences between different light regimes in the same month.
In contrast to the clear reduction in precocious males observed under LL conditions during the first annual cycle of life this study, ${ }^{11,20,21}$ the LL treatment had no effect on gonadal maturation during the second annual life cycle of the animals. Consecutive administration of LL 17 treatments (T1 and T2) in year two failed to influence the phase of the spermiation rhythm as compared to NP, and the rate of running males increased along with the seasonal profile of GSI (T1), which peaked in February, a crucial month in terms of the reproductive performance of this species. ${ }^{9}$ However, significantly lower percentages of running males and lower GSI values were observed in LL groups, suggesting that LL regimes administered throughout the most sensitive photolabile period during the first and second annual cycles reduced the amplitude of the spermiation rhythm with respect to the maximal levels attained under NP. On the other hand, when the rate of spermiation for the NP group was compared to that of the LLA-O (LLJO-AO) group (T2), no differences were found in the time when males began spermiating during the second annual cycle. Sea bass milt obtained by means of a gentle abdominal massage was first registered in mid-November, and the highest number of males releasing sperm for the first time was observed in January, followed by a reduction at the end of February. From this moment on, males were able to continue spermiating until the end of May. Of note, the cumulative percentage of spermiating fish attained maximum levels in February, and was maintained for three more months. Accordingly, spermiation may last for as long as 6 months (i.e., from mid-November to May), with an average duration of 124-127 days in this study. These observations are within the range of values reported for European sea bass, which is a group synchronous spawner and has a rather long spawning season, with males actively spermiating for up to 5-6 months Carrillo et al. ${ }^{9}$ this study, which ensures the fertilization of gametes throughout the entire reproductive season.

Altogether, these data demonstrate that LL regimes do not drastically alter gonadal development in 2-year-old male sea bass, suggesting that adult males might have acquired a previous potential competence to reproduce that is independent of the photoperiod history, including 18 inhibitory treatments of gametogenesis. This provides further evidence of an endogenous mechanism responsible for reproduction in this species. ${ }^{9,15}$ In contrast, in 2-year-old Atlantic cod, continuous artificial lighting in combination with shading suppresses sexual maturation. ${ }^{8}$ This indicates that different fish species behave differently, and thus the efficacy of artificial day length treatments needs to be tested for each particular species. On the other hand, our study has also explored the effects of exposure to LP on delaying gonadal maturation and enhancing growth during the second annual cycle. The present results (T3) confirm those of Rodríguez et al., ${ }^{17}$ demonstrating that LP induces a delay in both the time of maximum spermiation and testicular growth. In the present study, LP altered the phase of the spermiation rhythm, which peaked from April on, and of GSI, which also reached maximum values in April, without affecting the amplitude of these rhythms. It should be noted that sperm quality was not evaluated in the present study, and this particular aspect would require more detailed investigation. Previous work on the application of constant long days during two consecutive years resulted in a reduction of fecundity and egg quality in female sea bass, as the result of a mismatch between inappropriate environmental cues (i.e., a long photoperiod and low temperature during the reproductive period) that altered the hormonal reproductive cascade. ${ }^{13,14}$ It is known that artificial environmental situations can cause a dysfunction affecting the synthesis of estrogen and the rates of vitellogenesis and atresia, resulting in reduced quality of the progeny. In this regard, nothing is known about the effect of the discrepancy between the photoperiod and the temperature on testicular steroidogenesis and 
sperm quality. More detailed studies are therefore needed to explore the effects of the relationship between photoperiod and temperature on these aspects. ${ }^{13,17}$

Finally, we observed that males kept under continuous light in year two had a significantly lower weight and length than control animals, as previously described for male sea bass kept under the same light conditions during the first year. ${ }^{11}$ On the other hand, Felip et al..$^{20}$ failed to find any differences in the evolution of somatic growth in 1-yearold animals that received NP or LL treatments. Thus, differences in growth among distinct populations illustrate that, in addition to the photoperiod, genetic differences and other environmental factors, such as temperature, may play a role and even interact with the photoperiod to affect growth performance. ${ }^{11,13,17}$ Nevertheless, the occurrence of a full gonadal development period in year two, coinciding with a period of reduced growth, might explain why fish size (at least in terms of length) in the LL groups is equal to or evenlarger than that of fish in the control group. These findings support the idea that LL treatments during year two do not impair the reproductive competence of animals that reach puberty, as the allocation of energy among maintenance, growth and reproductive tasks takes place. Interestingly, this study shows that continuous long-day exposure in year two (i.e. NPLP, LL-LP) results in a slight increase in growth, both in terms of weight and length, with respect to fish kept under NP-NP and LL-NP conditions, providing a potentially beneficial effect for fish farming. Moreover, our data show that the fish were larger $(\mathrm{GW})$ in $\mathrm{T} 2$ than in $\mathrm{T} 3$ or T1, and also exhibited higher condition factor values. This might be attributed to the fact that fish of different size must satisfy different demands (i.e., growth, gonadal development, swimming activity, etc.), which thus affects their corpulence. These findings are in line with previous studies that considered the effect of long-day regimes lasting two ${ }^{7}$ or three ${ }^{13}$ consecutive years on the enhancement of somatic growth in male sea bass. We have shown that, at the time of incipient gonadal development (year one), fish exposed to LP display a greater amplitude annual GSI profile than those exposed to NP, as well as a one-month delay in gonadal maturation. During full gonadal maturation (year two in this study), the annual GSI profile of LP fish was shifted by one month with respect to that of the NP group. This is associated with a phase delay and increased amplitude of the annual rhythms of some key reproductive hormones, such as the pituitary gonadotropin releasing hormone $(\mathrm{sbGnRH})$ and the plasma levels of luteinizing hormone (LH) and 11-ketotestosterone (11-KT) Carrillo et al. ${ }^{7}$ Altogether, these endocrine changes are likely to affect LP fish gonadal development, which occurs later than in NP fish, and as a result, potentially favor somatic growth. In summary, the present study, conducted by means of three independent trials on a laboratory scale, demonstrates that continuous light administered during year one over a 12-month period, or a shorter time interval that includes the sensitive photolabile period (September), significantly reduces precocious maturation in yearling European sea bass males. The shorter treatment may have the added advantage of reducing the stress provoked by long-term exposure to LL. In contrast, LL does not prevent maturation at year two, supporting the existence of an endogenous rhythm for reproduction in sea bass. Furthermore, the administration of LP in year two delays maturation (i.e., shifts the peaks for GSI and the percentage of spermiating males from March to April, regardless of the photoperiod treatment used in year one), modifies the annual rhythms of some key hormones ${ }^{7}$ and enhances the potential growth of fish Rodríguez et al. ${ }^{17}$ this study. We also examined the duration of spermiation and observed that fish may actively spermiate for up to 6 months (mid21November-May), with an average duration of 124127 days. Finally, the close associations between specific growth rate in terms of length and the rate of precocity, and specific growth rate in terms of weight and corpulence observed in this species might be considered to be biological performance indicators for reared stock. Accordingly, the effect of combining continuous and long lighting on the control of sexual maturation under indoor rearing conditions might have potential commercial applications, thus improving productivity and reducing farm operating costs. In this vein, further studies are needed to verify the appropriateness of environmental treatments in the transition from a laboratory setting to outdoor conditions.

\section{Acknowledgements}

The authors wish to thank C. Marín and X. Simó for their technical assistance in rearing and sampling the fish. The work of the authors in this article was supported by UE projects Q5RS2002-01801 (PUBERTIMING) and Grants AGL2006-04672 (PUBERCONTROL) and CSD2007-00002 (AQUAGENOMICS) from the Spanish Ministry of Science and Innovation (MICINN) and PROMETEO/2010/003 (Generalitat Valenciana). RR was supported by a FPI fellowship from the MICINN (Spain).

\section{Conflicts of Interset}

None.

\section{References}

1. Bromage N, Porter M, Randall C. The environmental regulation of maturation in farmed finfish with special reference to the role of photoperiod and melatonin. Aquaculture. 2001;197:63-98.

2. Hulata G. Genetic manipulations in aquaculture: a review of stock improvement by classical and modern technologies. Genetica. 2001;111(1):155-173.

3. Zanuy S, Carrillo M, Felip A, et al. Genetic, hormonal and environmental approaches for the control of reproduction in the European sea bass (Dicentrarchus labrax). Aquaculture. 2001;202(3-4):187-203.

4. Migaud H, Davie A, Taylor JF. Current knowledge on the photoneuroendocrine regulation of reproduction in temperate fish species. J Fish Biol. 2010;76(1):27-68.

5. Carrillo M, Zanuy S, Felip A, et al. Hormonal and environmental control of puberty in perciform fish. The case of sea bass. Trends in Comparative Endocrinology and Neurobiology: Annals of the New York Academy of Sciences. 2009;1163:49-59.

6. Taranger GL, Carrillo M, Schulz RW, et al. Control of puberty in farmed fish. General Comparative Endocrinology. 2010;165(3):483-515.

7. Carrillo M, Begtashi I, Rodríguez L, et al. Long photoperiod on sea cages delays timing of first spermiation and enhances growth in male European sea bass (Dicentrarchus labrax). Aquaculture. 2010;299(14):157-164

8. Cowan M, Davie A, Migaud H. The effect of combining shading and continuous lighting on the suppression of sexual maturation in outdoorreared Atlantic cod, Gadus morhua. Aquaculture. 2011;320(1-2):113122

9. Carrillo M, Zanuy S, Prat F, et al. Sea bass (Dicentrarchus labrax). In: Broodstock Management and Egg and Larval Quality by Bromage NR \& Roberts RJ (Eds.), Blackwell Scientific Publications, Oxford, UK 1995;pp.138-168.

10. Saillant E, Fostier A, Menu B, et al. Sexual growth dimorphism in sea bass Dicentrarchus labrax. Aquaculture. 2001;202:371-387.

11. Begtashi I, Rodríguez L, Molés G, et al. Long-term exposure to continuous light inhibits precocity in juvenile male European sea bass (Dicentrarchus labrax, L.). I. Morphological aspects. Aquaculture. 2004;241:539-559. 
12. Felip A, Zanuy S, Carrillo M. Comparative analysis of growth performance and sperm motility between precocious and nonprecocious males in the European sea bass (Dicentrarchus labrax, L.) Aquaculture. 2006;256:570-578.

13. Zanuy S, Carrillo M, Ruiz F. Delayed gametogenesis and spawning of seabass (Dicentrarchus labrax L.) kept under different photoperiod and temperature regimes. Fish Physiology and Biochemistry. 1986;2(14):53-63.

14. Zanuy S, Prat F, Carrillo M, et al. Effects of constant photoperiod on spawning and plasma 17b-oestradiol levels of sea bass (Dicentrarchus labrax). Aquatic Living Resources 8. 1995;147-152.

15. Prat F, Zanuy S, Bromage N, et al. Effects of constant short and long photoperiod regimes on the spawning performance and sex steroid levels of female and male sea bass. Journal of Fish Biology. 1999;54(1):125137.

16. Rodríguez L, Begtashi I, Zanuy S, et al. Changes in plasma levels of reproductive hormones during first sexual maturation in European male sea bass (Dicentrarchus labrax L.) under artificial day lengths. Aquaculture. 2001a;202:235-248.

17. Rodríguez L, Zanuy S, Carrillo M. Influence of day length on the age at first maturity and somatic growth in male sea bass (Dicentrarchus labrax, L.). Aquaculture. 2001b 196:159-175.
18. Rodríguez L, Carrillo M, Sorbera LA, et al. Effects of photoperiod on pituitary levels of three forms of $\mathrm{GnRH}$ and reproductive hormones in the male European sea bass (Dicentrarchus labrax, L.) during testicular differentiation and first testicular recrudescence. Gen Comp Endocrinol. 2004;136(1):37-48.

19. Rodríguez L, Begtashi I, Zanuy S, et al. Long-term exposure to continuous light inhibits precocity in European male sea bass (Dicentrarchus labrax, L.): hormonal aspects. General Comparative Endocrinology. 2005;140:116-561.125.

20. Felip A, Zanuy S, Muriach B, et al. Reduction of sexual maturation in male Dicentrarchus labrax by continuous light both before and during gametogenesis regimes. Aquaculture. 2008;275:347-355.

21. Rodríguez R, Felip A, Cerqueira V, et al. Identification of a photolabile period to reduce sexual maturation in juvenile male sea bass (Dicentrarchus labrax) by continuous light regime. Aquaculture International. 2012;20(6):1071-1083.

22. Barnabé G. The sea bass. In: Production of aquatic animals CE Nash \& AJ Novotny (Eds.), Elsevier, Oxford, UK. 1995;pp.269-287.

23. Sokal RR, Rohlf FJ. Biometry. The principles and practice of statistics in biological research (2nd edn), W.H. Freeman, USA. 1981;pp.859.

24. Scott AP, Baynes SM, Skarphédinsson O, Bye VJ. Control of spawning time in rainbow trout, Salmo gairdneri, using constant long day lengths. Aquaculture. 1984;43:225-233. 